\title{
$\alpha$-defensin 1-3 and $\alpha$-defensin 4 as predictive markers of imatinib resistance and relapse in CML patients
}

\author{
Gabriel Etienne ${ }^{\mathrm{a}, \mathrm{b}}$, Maryse Dupouy ${ }^{\mathrm{a}}$, Patricia Costagliolic ${ }^{\mathrm{c}}$, Claudine Chollet $^{\mathrm{d}}$, Valérie Lagarde ${ }^{\mathrm{a}}$, \\ Jean-Max Pasquet ${ }^{\mathrm{a}}$, Josy Reiffers ${ }^{\mathrm{b}}$, Bertrand Garbay ${ }^{\mathrm{c}}$, François-Xavier Mahon ${ }^{\mathrm{a}, \mathrm{d}}$ and Béatrice Turcq ${ }^{\mathrm{a}, *}$ \\ ${ }^{a}$ Laboratoire Hématopoïese Leucémique et Cibles Thérapeutiques, INSERM U876, Université Victor Segalen \\ Bordeaux 2, Bordeaux, France \\ ${ }^{\mathrm{b}}$ Institut Bergonié, Centre Régional de Lutte Contre le Cancer de Bordeaux et du Sud-Ouest, Bordeaux, France \\ ${ }^{\mathrm{c}}$ Université de Bordeaux, IPB, EA 4135, ENSTBB, Bordeaux, France \\ ${ }^{\mathrm{d}}$ Laboratoire Hématologie, Hôpital Haut-Lévêque, CHU de Bordeaux, Bordeaux, France
}

\begin{abstract}
Objective: Imatinib mesylate is a tyrosine kinase inhibitor used as first line treatment in chronic myeloid leukaemia. Despite a remarkable effectiveness, treatment failure cases have been reported in 20 percent of CML patients. The identification of biomarkers which can predict the response to imatinib is our point of interest.

Methods: Gene expression profiling microarray was carried out on secondary imatinib resistant patients. Longitudinal studies were performed on imatinib treated responder/resistant patients. Then, Q-RT/PCR studies were realized on patients prior imatinib initiation.

Results: For imatinib responder patients, we observed a strong and lasting decrease of $\alpha$-defensin $1-3$ and $\alpha$-defensin 4 expression. For relapse patients, we observed a dramatic increase of $\alpha$-defensin 1-3 and $\alpha$-defensin 4 expression before BCR-ABL transcript increase. Moreover, before imatinib initiation, $\alpha$-defensin 1-3 and $\alpha$-defensin 4 expression was significantly lower in the resistant group than in the responder group.

Conclusion: The variation of expression of $\alpha$-defensin 1-3 and $\alpha$-defensin 4 in peripheral blood is associated with imatinib resistance and may reflect an adequate immune control of the disease. Monitoring of $\alpha$-defensin 1-3 and $\alpha$-defensin 4 could be helpful to predict the patients who are not going to respond to the treatment.
\end{abstract}

Keywords: Chronic myeloid leukaemia, imatinib resistance, $\alpha$-defensin, predictive biomarker

\section{Introduction}

Chronic Myeloid Leukemia (CML) is a clonal hematologic malignant disease of the hematopoietic stem cell in which a $\mathrm{t}(9 ; 22)$ ( $\mathrm{q} 34 ; \mathrm{q} 11)$ reciprocal translocation leads to a 22 abnormal chromosome designated Philadelphia chromosome $(\mathrm{Ph})$. Translocation fus-

\footnotetext{
* Corresponding author: Béatrice Turcq, Ph.D., Laboratoire Hématopoï̀se Leucémique et Cibles Thérapeutiques, Inserm U876, Université Victor Segalen Bordeaux 2, 146 rue Léo Saignat, 33076 Bordeaux Cedex, France. Tel.: +33 557571 051; Fax: +33 556938 883; E-mail: beatrice.turcq@u-bordeaux2.fr.
}

es BCR and ABL genetic sequences resulting in the BCR-ABL hybrid gene coding the fusion protein BCRABL. This constitutively activated tyrosine kinase protein is responsible for the transformation of BCR-ABL cells $[4,13]$. Recently, novel therapeutic molecules have been used to treat CML patients. Imatinib mesylate (STI571, Glivec, Gleevec) is a tyrosine kinase inhibitor that blocks the BCR-ABL kinase activity and selectively eradicates CML cells [11]. The International Randomized Study of Interferon and Imatinib (IRIS) defines imatinib as the major therapeutic agent for the treatment of patients with CML [7].

Imatinib can induce cytogenetic remissions, com- 
plete or nearly complete, in up to 80 percent of patients when they are treated in chronic phase $(\mathrm{CP})$. However, in 20 percent of CML patients, treatment is inefficient. There are two kinds of resistance: either patients never respond to treatment (primary resistance), or patients respond and, after months or years, escape (secondary resistance) [16]. The frequency of BCR-ABL mutations in imatinib resistant patients ranges from $40 \%$ to $90 \%$, depending on the definition of resistance. But in some patients, no mutation allows to explain the resistance phenomenon.

Since alternative therapeutic options are available, identification of additional predictive factors of imatinib resistance is pertinent. Up to now, prognostic factors used to determine imatinib response are: Sokal risk scores including clinical parameters [17], the phase of the disease (chronic or accelerated), and the fact that other therapies were administered prior to imatinib [18].

In this study, we focused on the identification of specific early predictive biomarkers of imatinib resistance. To this aim, we performed pangenomic microarray analysis on CML patients with secondary imatinib resistance. Two genes encoding $\alpha$-defensin 1-3 (DEFA1-3) and $\alpha$-defensin 4 (DEFA4) were highly expressed at the time of imatinib resistance. Defensins are small antimicrobial peptides involved in innate and adaptive defense systems [8]. They are synthesized as preprodefensins in the bone marrow precursors of blood granulocytes, and the mature defensins are stored in the granules of neutrophils. Interestingly, $\alpha$ - and $\beta$-defensins have already been described as putative biomarkers in different cancers $[1,5,10,14]$. We thus investigated the expression of DEFA1-3 and DEFA4 on imatinib responder and resistant CML-CP patients. Our data suggests that the variation of expression of DEFA1-3 and DEFA4 is associated with imatinib resistance, while a low level of DEFA1-3 and DEFA4 in CML patients before imatinib treatment is a predictive factor for resistance.

\section{Materials and methods}

\subsection{Patients}

All patients enrolled in this study provided informed consent according to the Declaration of Helsinki. CML-CP patients treated with imatinib standard dose (400 mg/day) (Novartis, Basel, Switzerland) were considered for this study. Primary resistance to ima- tinib was defined as lack of cytogenetic response after at least six months of imatinib therapy. Secondary resistance was defined as loss of previous, at least partial, cytogenetic response.

Microarray analysis was performed on two secondary resistant patients. Quantitative polymerase chain reaction during therapy was performed on 25 responders and 7 secondary resistant patients. Quantitative polymerase chain reaction before imatinib treatment was performed on 41 responders and 21 resistant patients.

\subsection{Microarray analysis}

Total RNA from leucocytes was extracted using Trizol reagent method (Invitrogen), and purified further using RNeasy Mini Kit (Qiagen). DNA contaminants were removed by DNase I treatment (Ambion). RNA concentration was determined by $\mathrm{OD}_{260}$. From each sample, 500 ng RNA was then used to prepare the Cy3 and Cy5-labelled probes with a Low RNA Input Linear Amplification kit (Agilent Technologies). The incorporation of the $\mathrm{Cy} 3$ and $\mathrm{Cy} 5$ fluorescent dyes was verified with a spectrophotometer NanoDrop ND-1000. Hybridization and washing of the $22 \mathrm{~K}$ human Agilent Microarrays were performed as recommended by the manufacturer. For the microarrays scanning, we used a Dual Laser Microarray Scanner (Agilent Technologies). Spot intensities were then quantified with Agilent Feature Extraction software version A.7.5.1. The settings were as follows: Spot analysis method using Cookie Cutter, pixel outlier rejection based on IQR, non-uniformity outlier flagging on, population outlier flagging on, no background subtraction, spatial detrend on, normalization feature selection by using rank consistency filter, multiplicative errors of 0.15 for red and 0.25 for green, and additive errors of 20 for both red and green channels. Using a rank consistency filter, features were subjected to a combination linear and LOWESS normalization algorithm (www.agilent.com). Quality control algorithms in the software detected poor quality spots that were excluded from analysis and contained a non zero value for any of the following fields: IsSaturated, IsFeatNonUnifOL, IsBGNonUnifOL, IsFeatPopnOL, IsBGPopnOL and IsManualFlag. For each gene sequence present on the microarrays, statistical significance of differential gene expression was determined by calculating $\mathrm{p}$-Values according to the following equation:

$$
p \text { Value }=1-\operatorname{Erf}\left(\frac{|x d e v|}{\sqrt{2}}\right)
$$


Where Erf is the error function for a Gaussian distribution of zero mean and

$$
x d e v=\frac{\text { LogRatio }}{\text { LogRatioError }}
$$

The differentially expressed genes were defined as: (1) the absolute value of the $\mathrm{Cy} 5 / \mathrm{Cy} 3 \log 10$ was more than 0.4 (the variation of gene expression was more than 2.5 fold), (2) either Cy3 or Cy5 processed signal value was more than 1500 and (3) the LogRatio p-Value $<0.002$.

\subsection{Plasmids}

The DEFA1-3, DEFA4, $\beta$ ACT PCR fragments, generated using DEFA 1-3 forward primer 5'-AGGCTCAA GGAAAAACATGG-3' and reverse primer 5'-GCAGA ATGCCCAGAGTCTTC-3', DEFA4 forward primer 5'-GCAGCTGAGCTTGCAGAATA-3' and reverse primer 5'-GGACAAAGTATAGGAGAAACAACCA-3', ACT forward primer 5'-AGCATCGGGT GATGTTCA TT-3' and reverse primer 5-ATTACAAGCATGCGTC ACCA-3' were inserted into the pCR ${ }^{\circledR}$ 2.1-Topo vector using the TOPO TA Cloning ${ }^{\circledR}$ kit (Invitrogen). To produce standard curves, these plasmids were used as template DNA at concentrations ranging from $10^{8}$ to $10^{1}$ copies $/ \mu 1$

\section{4. $Q-R T / P C R$}

cDNA was synthesized from $1 \mu \mathrm{g}$ of total RNA with random hexamers in a final volume of $20 \mu \mathrm{l}$ (Roche). Quantitative polymerase chain reaction (Q-RT/PCR) was carried out in 96-well ABgene plates using the Mx3005P system (Stratagene) with the SYBR ${ }^{\circledR}$ Green Master Mix reaction (Stratagene). All reactions were performed in a total volume of $25 \mu \mathrm{l}$ and contained $2 \mu \mathrm{l}$ of cDNA and $6.25 \mu \mathrm{M}$ of each primer set. Each sample was analyzed in triplicate. Negative controls without added reverse transcriptase were performed. The primer sequence information is: DEFA1-3 forward primer 5'-AGGCTCAA GGAAAAACATGG-3' and reverse primer 5'-GCAGAATGCCCAGAGTCTTC-3', DEFA4 forward primer 5'-GCAGCTGAGCTTGCAG AATA-3' and reverse primer 5'-GGACAAA GTATA GGAGAAACAACCA-3', ACT forward primer 5' AGCATCGGGTGATG TTCATT-3' and reverse primer 5-ATTACAAGCATGCGTCACCA-3'. DEFA1-3 primers amplify DEFA1, DEFA2 and DEFA3. Thermal cycling was performed at $95^{\circ} \mathrm{C}$ for $10 \mathrm{~min}$ followed by 40 cycles comprising each a denaturation step at $95^{\circ} \mathrm{C}$ for $30 \mathrm{~s}$, and an annealing/extension step at $58^{\circ} \mathrm{C}$ for $45 \mathrm{~s}$. Amplification of the appropriate product was verified by continuous monitoring fluorescence through the dissociation temperature of the PCR product at a temperature transition rate of $0.1^{\circ} \mathrm{C} / \mathrm{s}$ to generate a melting curve. DEFA1-3 transcript levels were expressed as a ratio to the $\beta$ actin control transcripts or expressed as a ratio to the $\mathrm{ABL}$ control transcripts.

BCR-ABL mRNA transcript levels were quantified as previously described [3].

\subsection{Statistical analysis}

Statistical analysis was carried out using Student's t-test.

\section{Results}

To search predictive biomarkers for imatinib resistance in CML, transcriptomic analysis were performed on secondary resistant patients, whatever are their mutational status. For a given patient, microarray analyses were performed at the time of response and relapse. Comparison between the response and relapse is done independently for each patient to avoid genetic variation between patients.

BCR-ABL mRNA transcript level and blood count similarity, in both conditions, was a criterion to select response and relapse samples. Thus, the result was not influenced by difference in BCR-ABL expression. Two patients (P1 and P2) with secondary imatinib resistance, still in complete hematological response at the time of analysis, filled these conditions (Fig. 1). Analysis using Agilent 22K human 60-mer oligonucleotide pan genomic microarray (Agilent technologies) was performed. Eighteen down-regulated genes and 4 upregulated genes were differentially expressed (greater than-2.5-fold change in expression levels with a p-value less than 0.001). Two genes were highly deregulated at the time of imatinib resistance: the DEFA1-3 and DEFA4 genes (Table 1). DEFA1-3 and DEFA4 expression was 92 and 24 times higher for relapsing patient 1 , DEFA1-3 and DEFA4 expression was 14 and 5 times higher for relapsing patient 2 . The over-expression of both genes was confirmed by Q-RT/PCR in the same samples. DEFA1-3 expression of relapsing patients was about 3000 (P1) and 27 (P2) times higher than responding patients, and DEFA4 expression was 420 (P1) and 20 (P2) times higher. Therefore, our data obtained 

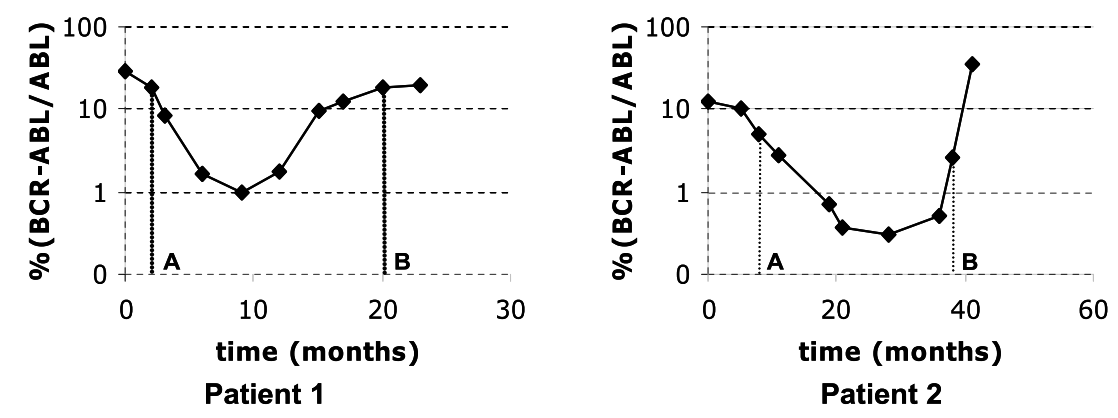

Fig. 1. Kinetic of BCR-ABL expression in two secondary resistant patients (Patient1, Patient2) used for microarray analyses. BCR-ABL transcripts measured by Q-RT/PCR and expressed as a ratio to the ABL control transcripts x 100\%. A and B represent chosen samples when patients respond and when patients relapse respectively.

Table 1

Comparison of results of DEFA1-3 and DEFA4 expression obtained by microarray and QRT/PCR experiments

\begin{tabular}{llcc}
\hline & & \multicolumn{2}{c}{ Ratio B/A } \\
\cline { 3 - 4 } & & DEFA1-3 & DEFA4 \\
\hline Microarray & Patient 1 & 92 & 24 \\
& Patient 2 & 14 & 5 \\
Q-RT/PCR & Patient 1 & 3532 & 421 \\
& Patient 2 & 27 & 20 \\
\hline
\end{tabular}

Patient 1, patient 2, samples A and B are described in Fig.1. Ratio B/A is expression of DEFA1-3 or DEFA4 when the patients relapse (B) compared when the patients respond (A).

by Q-RT/PCR method confirm the trend observed after the microarray analysis.

Since over-expression of DEFA1-3 seemed to be associated with secondary imatinib resistance, prospective analysis of DEFA1 expression in CML-CP during therapy was performed. Samples from 25 responders and 7 secondary resistant patients were analyzed by QRT/PCR for DEFA1-3 and BCR-ABL expressions. Kinetics of BCR-ABL and DEFA1-3 expression were performed on these patients. Two control genes were used in the DEFA1-3 Q-RT/PCR experiments. An example of kinetics of BCR-ABL and DEFA1-3 expression in two imatinib responders is shown Fig. 2(A, B).

For imatinib treated patients, the BCR-ABL and DEFA1-3 expressions quickly decrease. In all imatinib responders we observed a long-lasting DEFA1 mRNA transcript level decrease, with a DEFA $1-3$ to $\beta$-actin ratio stabilized around $10^{3}-10^{4}$ and a DEFA1-3 to ABL ratio stabilized around 10. BCR-ABL and DEFA1-3 expression decrease are parallel. An example of BCRABL and DEFA1-3 kinetics expression in two imatinib resistant patients is shown in Fig. 2(C, D). For patients who became resistant to imatinib, the low level of expression of DEFA1-3 was followed by a dramatic increase after a few months. Interestingly, the increase in DEFA1-3 transcripts occurred at a time when BCRABL mRNA transcript levels were still decreasing. The median duration of time between the DEFA1-3 expression increase and BCR-ABL mRNA transcript level increase was 6 months (range from 0 to 23 months), strongly suggesting that the variation of DEFA1-3 expression may not be correlated with that of BCR-ABL. Kinetics of BCR-ABL and DEFA1-3 expression in 23 imatinib responders and 5 resistant patients are shown in supplemental figure. Imatinib treated CML patients kinetics analyses allowed us to identify DEFA1-3 as candidate marker for early relapse identification.

Interestingly, besides being a good marker of the apparition of a resistant phenotype toward imatinib, DEFA1-3 and DEFA4 transcript levels may be used to discriminate between responder and resistant patients before imatinib treatment. Using our sample collection, we were able to measure the DEFA1-3 transcript levels in samples from imatinib responder $(n=41)$ and imatinib resistant $(n=21)$ patients that were collected before initiation of the imatinib treatment (Fig. 3A). For responder patients DEFA1-3/ $\beta$-actin expression mean and median were $3.8 \times 10^{6}$ and $3.3 \times 10^{5}$, respectively. For resistant patients, DEFA1-3/ $\beta$-actin expression mean and median were $5 \times 10^{4}$ and $2.1 \times 10^{4}$, respectively. Statistical Student's t-test carried on these two populations showed significant difference between responders versus resistant patients with a p-value $<$ 0.0001 . DEFA4 transcript levels were also performed (Fig. 3B). For responder patients DEFA $4 / \beta$-actin expression mean and median were $5.9 \times 10^{4}$ and 4.3 $\times 10^{3}$, respectively. For resistant patients, DEFA $4 / \beta$ actin expression mean and median were $9.6 \times 10^{2}$ and $2.7 \times 10^{2}$, respectively. Statistical Student's t-test carried on these two populations showed significant difference between responders versus resistant patients with a p-value $<0.0001$. Therefore, DEFA1-3 and 
A

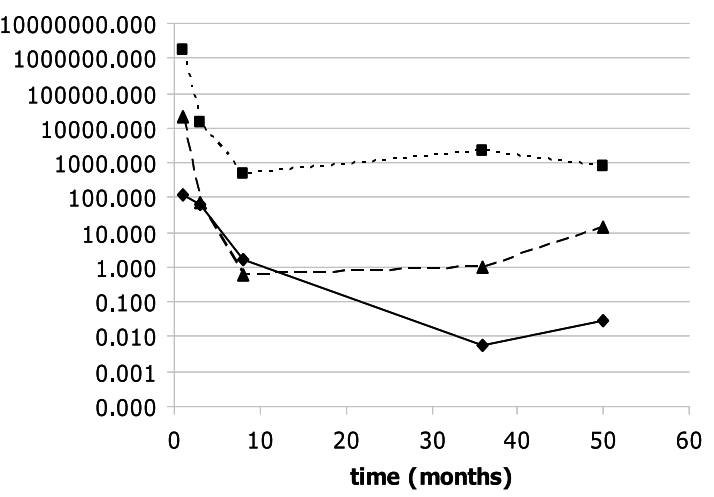

C

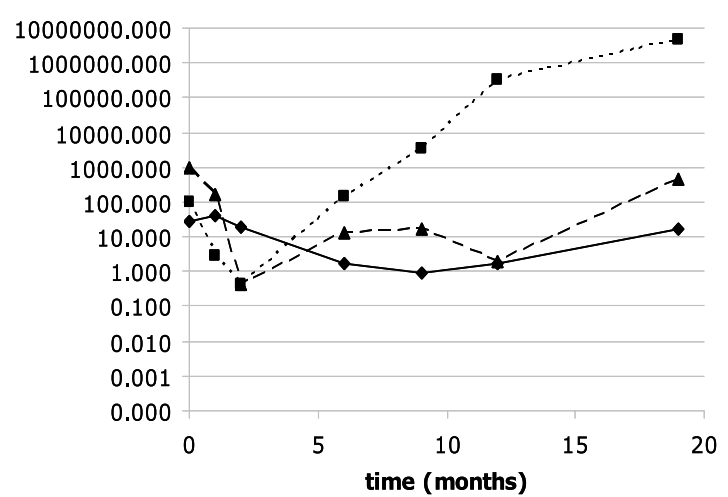

$\mathrm{B}$

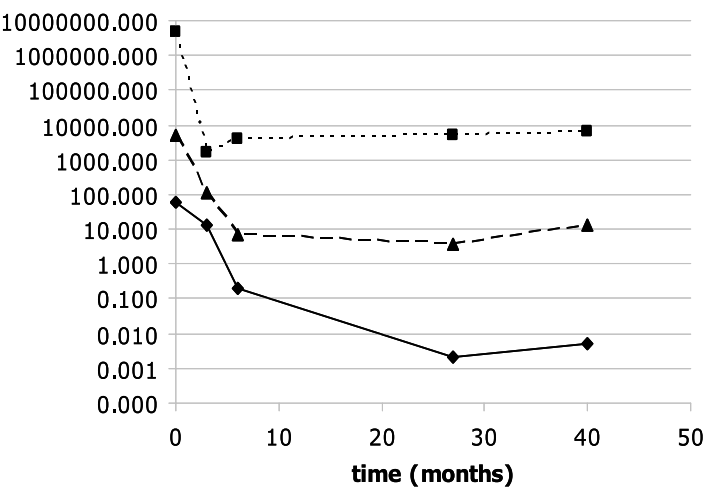

D

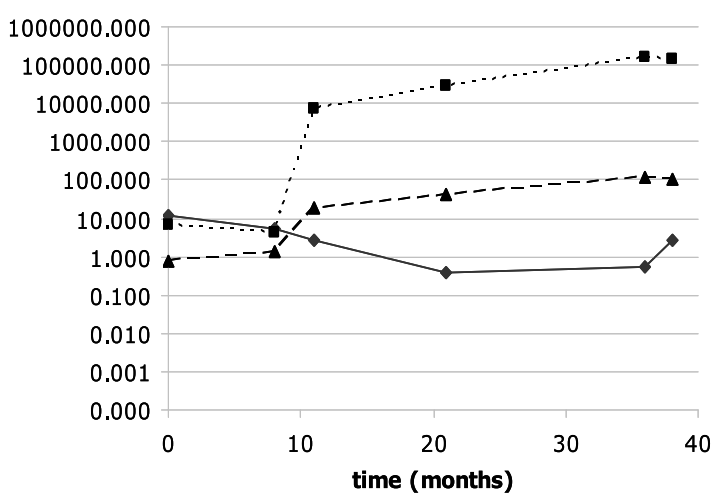

Fig. 2. Kinetics of BCR-ABL and $\alpha$-defensin 1-3 expression in 4 representative patients. For each patient, the kinetic of BCR-ABL transcripts measured by Q-RT/PCR (expressed as a ratio to the ABL control transcripts x 100\%) is represented by the bold black lines, the kinetic of the $\alpha$-defensin 1-3 transcripts measured by Q-RT/PCR expressed as a ratio to the $\beta$ actin control transcripts is illustrated by the dotted lines and the kinetic of the $\alpha$-defensin 1-3 transcripts measured by Q-RT/PCR expressed as a ratio to the ABL control transcripts is illustrated by the dashed lines. A and $\mathrm{B}$ represent two imatinib responders. $\mathrm{C}$ and $\mathrm{D}$ represent two imatinib resistant patients.

DEFA4 mRNA level in peripheral blood cells can be used to identify CML patients who are at risk of being imatinib-resistant, before initiation of the therapy.

\section{Discussion}

Previous studies using transcriptomic analyses have pointed out variation of $\alpha$-defensin expression in CML patients. Nowicki et al. [15] reported down-regulated expression of DEFA1 and DEFA4 in CML-CP cells in comparison to their normal counterparts. Hagberg et al. [9] showed higher expression of DEFA4 prior to interferon alpha therapy in interferon alpha resistant patients but the difference between the two groups in DEFA4 expression was not found to be significant using Q-RT/PCR. Although the significance of such vari- ations remains unclear, our results suggest a particular role of $\alpha$-defensins in CML.

Using Q-RT/PCR, we investigated DEFA1-3 expression in various haematological disorders, prior therapy, including acute myeloid leukaemia, myelodysplasia, Philadelphia-negative myeloproliferative syndromes, CML and healthy donors. DEFA1-3 expression was highest in CML patients with a mean DEFA1$3 / \beta$-actin ration of $10^{6}$ copies whereas in other malignancies or healthy donors this was found to be $10^{3}$ or less (data not shown). Defensins are small peptides involved in innate and adaptative host defense mechanisms. Two classes of defensins have been identified, alpha and beta, that differ with respect to their structure and tissue pattern of expression. They are synthesized as prodefensins in the bone marrow precursors of blood granulocytes and mature defensins are 

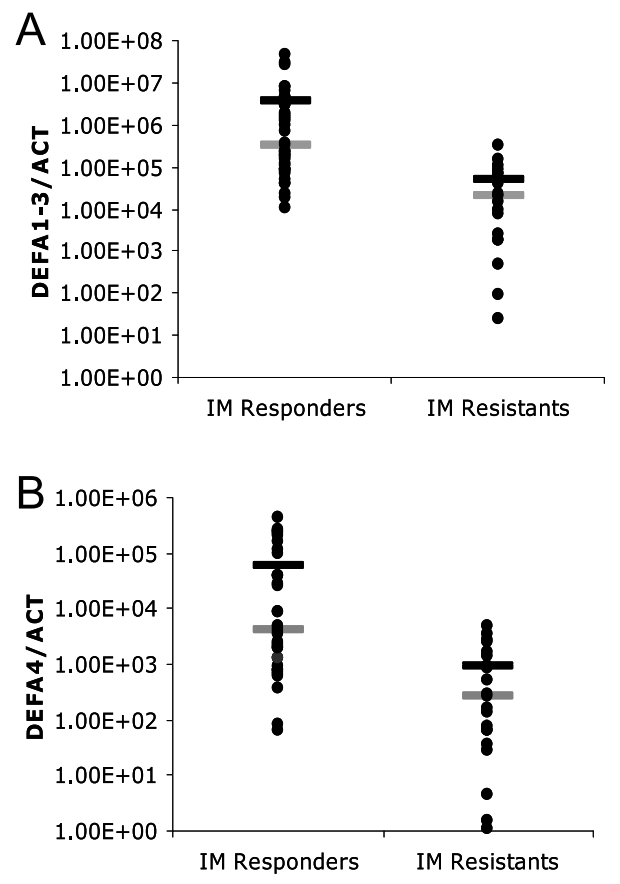

Fig. 3. Comparison of $\alpha$-defensin 1-3 and $\alpha$-defensin 4 expression at day 0 imatinib in both imatinib (IM) responder and resistant population. Black and grey dashes indicate mean and median values respectively. Defensin expression calculated as the ratio [ $\alpha$-defensin copy number/ $\beta$ actin copy number]. Level of significance set at $0.01 \%$. The statistical power of the test is $99 \%$.

stored in the granules of neutrophils. By cell sorting on a CML patient, we verified as described by Klotman and Chang that the highest level of DEFA expression is found in neutrophils [12]. Moreover, for the patients where we had the blood formula, we did not establish a correlation between the level of DEFA expression and the quantity of neutrophils. Recently, Droin et al. have shown a new property of DEFA1-3 peptides in chronic myelomonocytic leukemia cells: high concentration of DEFA1-3 peptides inhibit differentiation of peripheral blood monocytes into macropahages and contribute to the accumulation of monocytes [6]. Numerous studies have pointed out the crucial role of defensin in linking innate immunity with the adaptative immune system [19].

As variation of DEFA1-3 expression appeared not to be correlated with that of BCR-ABL one, we hypothesized that the relatively high expression of DEFA1-3 prior the imatinib treatment may reflect an adequate immune control of the disease, allowing a long-lasting response to imatinib. A putative role of defensin in tumor suppression was highlighted by Bullard et al. [2], for the $\beta$-defensin 1 in prostate cancer. This putative tumor-suppressor effect of $\alpha$-defensin 1 could be nec- essary for the efficacy of the imatinib treatment. We speculate that the low expression of defensin prior imatinib treatment interferes with immune system and contributes to the non response.

In addition, results obtained on the follow-up of responders and secondary resistant patients showed that an increase in DEFA1-3 expression took place before the increase in BCR-ABL, thereby allowing to predict secondary resistant patients relapse. However, we cannot explain why DEFA1-3 expression increases for relapse patients while high DEFA1-3 expression before imatinib treatment is a sign of good response to imatinib.

In conclusion, the monitoring of the expression of DEFA1-3 allows the prediction of early relapse in CML patients. Moreover, we have demonstrated that quantification of DEFA1-3 and DEFA4 mRNAs may be helpful to identify patients which may not respond to imatinib, and thus will help the medical staff to propose them alternative therapeutic options.

\section{Acknowledgments}

This work was supported by grants from Ligue Nationale contre le Cancer (Comité des Landes et de Dordogne, France), Fondation de France (Paris, France) and the Association of Laurette Fugain (Paris, France).

\section{Supplemental material}

Supplemental material can be obtained by request from the corresponding author, at: beatrice.turcq@ubordeaux 2. fr.

\section{References}

[1] Y. Arimura, J. Ashitani, S. Yanagi, M. Tokojima, K. Abe, H. Mukae et al., Elevated serum beta-defensins concentrations in patients with lung cancer, Anticancer Research 24 (2004), 4051-4057.

[2] R.S. Bullard, W. Gibson, S.K. Bose, J.K. Belgrave, A.C. Eaddy, C.J. Wright et al., Functional analysis of the host defense peptide Human Beta Defensin-1: new insight into its potential role in cancer, Molecular Immunology 45 (2008), 839-848.

[3] M. Colombat, M.P. Fort, C. Chollet, G. Marit, C. Roche, C. Preudhomme et al., Molecular remission in chronic myeloid leukemia patients with sustained complete cytogenetic remission after imatinib mesylate treatment, Haematologica 91 (2006), 162-168.

[4] G.Q. Daley, R.A. Van Etten and D. Baltimore, Induction of chronic myelogenous leukemia in mice by the P210bcr/abl gene of the Philadelphia chromosome, Science 247 (1990), $824-830$. 
[5] N. Droin, J.B. Hendra, P. Ducoroy and E. Solary, Human defensins as cancer biomarkers and antitumour molecules, Journal of proteomics 72 (2009), 918-927.

[6] N. Droin, A. Jacquel, J.B. Hendra, C. Racoeur, C. Truntzer, D. Pecqueur et al., Alpha-defensins secreted by dysplasic granulocytes inhibit the differentiation of monocytes in chronic myelomonocytic leukemia, Blood 115 (2010), 78-88.

[7] B.J. Druker, F. Guilhot, G. O'Brien, I. Gathmann, H. Kantarjian, N. Gattermann et al., Five-year follow-up of patients receiving imatinib for chronic myeloid leukaemia, New England Journal of Medicine 355 (2006), 2408-2417.

[8] T. Ganz, M.E. Selsted and R.I. Lehrer, Defensins, European Journal of Haematology 44 (1990), 1-8.

[9] A. Hagberg, U. Olsson-Stromberg, U. Wickenberg-Bolin, H. Göransson, A. Isaksson, M. Bengtsson et al., Gene expression analysis identifies a genetic signature potentially associated with response to $\alpha$-IFN in chronic phase CML patients, Leukemia Research 31 (2007), 931-938.

[10] D.A. Holterman, J.I. Diaz, P.F. Blackmore, J.W. Davis, P.F. Schellhammer, A. Corica et al., Overexpression of alphadefensin is associated with bladder cancer invasiveness, Urologic Oncolology 24 (2006), 97-108.

[11] H. Kantarjian, C. Sawyers, A. Hochhaus, F. Guilhot, C. Schiffer, C. Gambacorti-Passerini et al., Hematologic and cytogenetic responses to imatinib mesylate in chronic myelogenous leukaemia, New England Journal of Medicine 346 (2002), $645-652$.

[12] M.E Klotman and T.L. Chang, Defensins in innate antiviral immunity, Nature Reviews Immunology 6 (2006), 447-456.

[13] J.V. Melo and M.W. Deininger, Biology of chronic myelogenous leukemia - signaling pathways of initiation and transformation, Hematology/Oncology Clinics of North America 18 (2004), 545-568.

[14] M.J. Nam, M.K. Kee, R. Kuick and S.M. Hanash, Identification of defensin alpha6 as a potential biomarker in colon adenocarcinoma, The Journal of Biological Chemistry 280 (2005), 8260-8265.

[15] M.O. Nowicki, P. Pawlowski, T. Fischer, G. Hess, T. Pawlowski and T. Skorski, Chronic myelogenous leukaemia molecular signature, Oncogene 22 (2003), 3952-3963.

[16] A. Quintás-Cardama, M. Hagop, H. Kantarjian and J.E. Cortes, Mechanisms of primary and secondary resistance to imatinib in chronic myeloid leukemia, Cancer Control 16 (2009), 122-131.

[17] J.E. Sokal, E.B. Cox, M. Baccarani, S. Tura, G.A. Gomez, J.E. Robertson et al., Prognostic discrimination in "good-risk" chronic granulocytic leukemia, Blood 63 (1984), 789-799.

[18] M. Talpaz, R.T. Silver, B.J. Druker, J.M. Goldman, C. Gambacorti-Passerini, F. Guilhot et al., Imatinib induces durable hematologic and cytogenetic responses in patients with accelerated phase chronic myeloid leukemia: results of a phase 2 study, Blood 99 (2002), 1928-1937.

[19] D. Yang, A. Biragyn, L.W. Kwak and J.J. Oppenheim, Mammalian defensins in immunity: more than just microbicidal, Trends in Immunology 23 (2002), 291-296. 


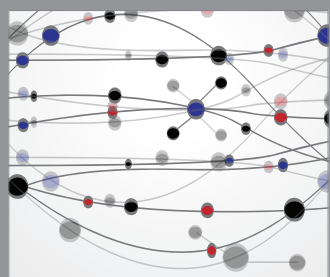

The Scientific World Journal
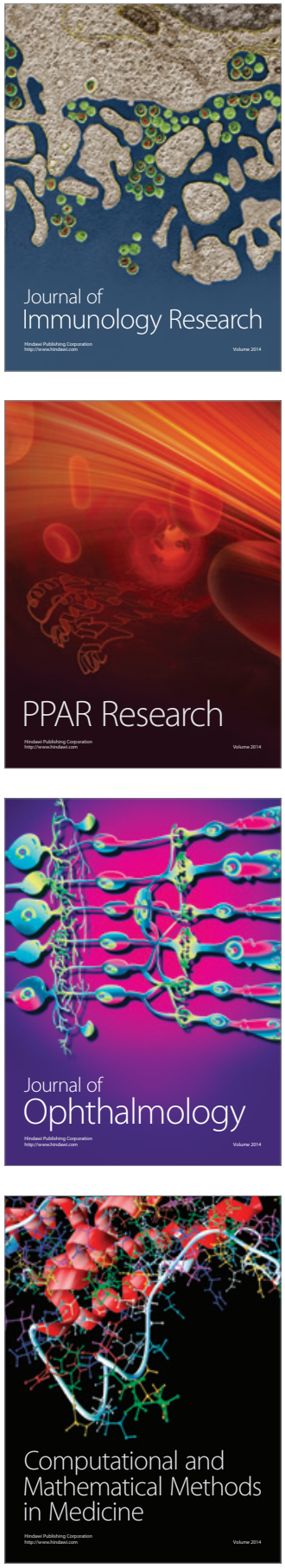

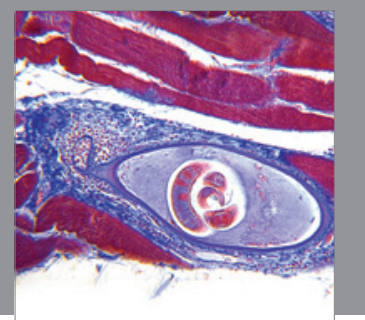

Gastroenterology

Research and Practice
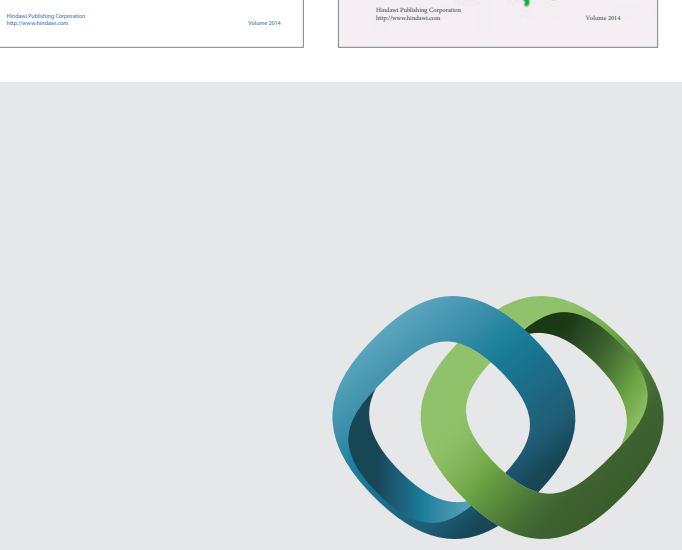

\section{Hindawi}

Submit your manuscripts at

http://www.hindawi.com
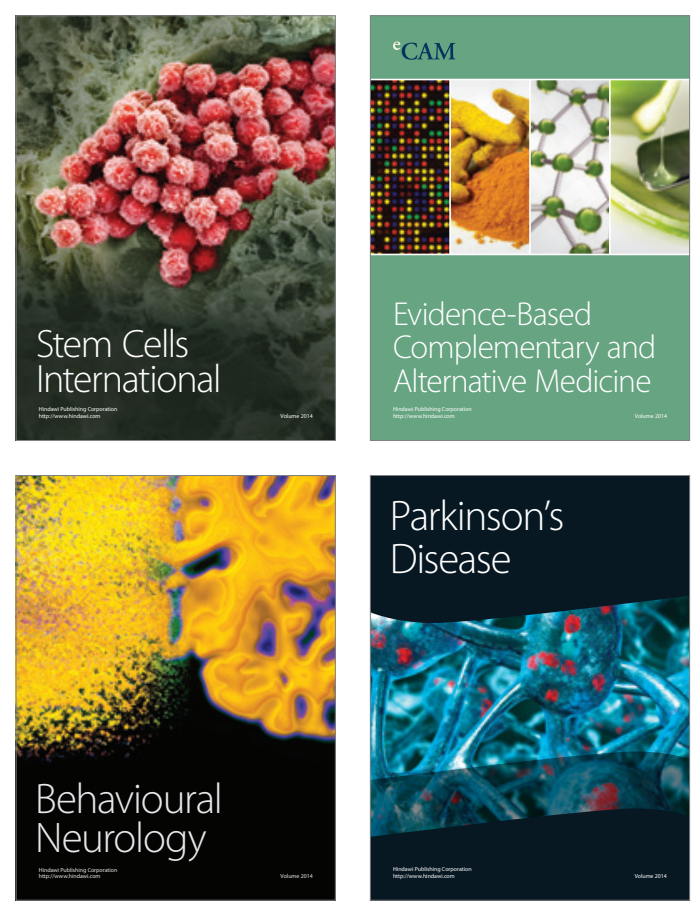

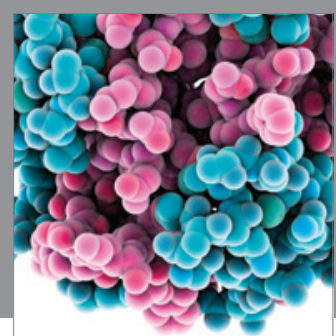

Journal of
Diabetes Research

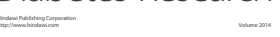

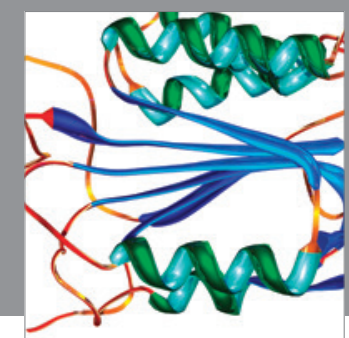

Disease Markers
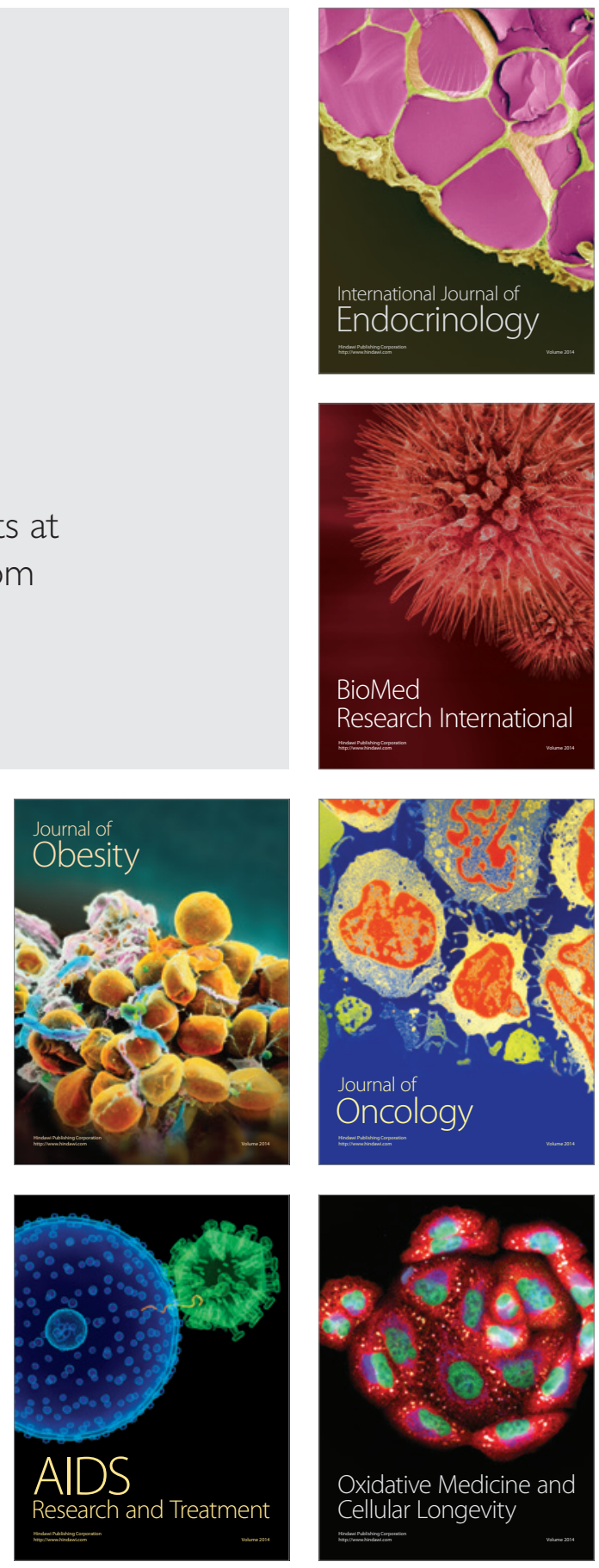